\title{
Éléments sur le système éducatif iranien
}

\section{Clotilde Reiss}

\section{OpenEdition}

Journals

Édition électronique

URL : http://journals.openedition.org/ries/590

DOI : 10.4000/ries.590

ISSN : 2261-4265

\section{Éditeur}

Centre international d'études pédagogiques

\section{Édition imprimée}

Date de publication : 1 décembre 2008

Pagination : 154-159

ISSN : 1254-4590

\section{Référence électronique}

Clotilde Reiss, «Éléments sur le système éducatif iranien », Revue internationale d'éducation de Sèvres

[En ligne], 49 | décembre 2008, mis en ligne le 01 décembre 2011, consulté le 19 avril 2019. URL : http://journals.openedition.org/ries/590 ; DOI : 10.4000/ries.590

Ce document a été généré automatiquement le 19 avril 2019

(c) Tous droits réservés 


\title{
Éléments sur le système éducatifiranien
}

\author{
Clotilde Reiss
}

1 L'Iran connaît l'émergence d'un système éducatif moderne et centralisé dans les années 1920-1930, suite à la prise de pouvoir du roi Reza Shah qui fonde la dynastie pahlavie en 1925. Ce roi entreprend une modernisation du pays sur le modèle de pays européens et une centralisation de l'administration du territoire iranien. En matière d'éducation, il initie le développement d'une école moderne aux dépens des écoles religieuses traditionnelles. L'État pahlavi prend progressivement le contrôle du système éducatif et encourage une sécularisation de l'enseignement. La République islamique, au pouvoir depuis 1979, prend le contrepied de cette tendance et choisit de recentrer l'éducation sur la religion. Si cette réforme de l'éducation incarne une rupture, elle s'inscrit aussi largement dans l'héritage du système scolaire iranien qui s'est construit au cours du XX ${ }^{e}$ siècle.

\section{L'émergence de l'école moderne centralisée}

2 Depuis l'islam médiéval, l'instruction se faisait dans des institutions religieuses sous le contrôle du clergé. Les maktab (« lieu d'écriture ») accueillaient traditionnellement les jeunes enfants âgés de cinq ou six ans et jusqu'à lâge de neuf ou dix ans. Placées sous l'autorité d'un mollah ${ }^{1}$ ou d'un professeur, elles étaient au XIX ${ }^{\mathrm{e}}$ siècle présentes dans la majorité des villages et assuraient un enseignement aux filles et aux garçons. L'enseignement se concentrait sur l'apprentissage de la langue persane ainsi que sur la mémorisation du Coran et de célèbres poèmes persans. L'apprentissage passait par la répétition et variait selon l'instruction du professeur. Les élèves les plus talentueux avaient la possibilité de poursuivre leurs études dans les madrasah, (« lieu d'étude ») où les études se concentraient sur les sciences religieuses. Cette formation débouchait sur l'obtention d'un diplôme en jurisprudence (en droit islamique) ou permettait à l'élève d'enseigner auprès de son maître-enseignant.

3 Les premières écoles modernes ne répondent pas d'une volonté de l'État mais sont nées de manière marginale, à l'initiative de particuliers. Leur caractère moderne repose sur le 
fait qu'elles valorisent la science et la raison (sur le modèle de la modernité occidentale). Les institutions pionnières sont les écoles missionnaires fondées dans les années 1830 . D'abord destinées à la minorité chrétienne du pays, elles se sont progressivement ouvertes aux musulmans iraniens. Puis ce sont des hommes d'État réformistes, pensant l'éducation comme un facteur de développement, qui encouragent la création d'écoles modernes. La première école supérieure moderne est ainsi créée en 1851 par le grand chancelier Amir Kabir sous le roi Nasseroldin Shah (1831-1896). Sur le modèle de l'École polytechnique, il fonde le prestigieux établissement Dar ol Founoun à Téhéran, qui assure des formations militaires, scientifiques et médicales. D'autres établissements apparaissent au XIX siècle à l'initiative d'intellectuels appartenant à la classe aisée et urbaine iranienne. Ces écoles, qui touchent une élite minoritaire, s'opposent à l'instruction traditionnelle et s'inspirent des modèles européens. Ces différentes initiatives tentent une sécularisation de l'instruction mais elles sont aussi le résultat d'un compromis nécessaire avec un milieu clérical hostile. L'instruction religieuse sera ainsi maintenue de manière obligatoire dans l'ensemble de ces établissements. À Dar Ol Founoun notamment, les différentes filières sont conçues pour ne pas totalement écarter les matières religieuses.

4 La révolution constitutionnelle de 1906 marque l'émergence d'un engagement de l'État dans l'établissement d'un système éducatif moderne. Les principes de la Constitution de 1906 instituent l'école moderne, son organisation ainsi que ses finalités. Selon l'article 19, les affaires scolaires sont placées sous la direction de l'État, et l'enseignement scolaire, ainsi que la création d'écoles, sont gérés par le ministère des sciences et des arts. Tous les établissements scolaires, modernes comme traditionnels, sont ainsi placés sous la tutelle de l'État. D'autres principes sont posés, tels que l'égal accès à l'éducation pour les filles et les garçons ou l'interdiction formelle des châtiments corporels jusqu'alors couramment pratiqués. Enfin, l'article 17 affirme le caractère obligatoire de l'école dès l'âge de sept ans. À partir de ce moment, les milieux religieux critiquent l'école centralisée car elle échappe à leur contrôle et offre un enseignement sécularisé dans lequel l'instruction religieuse est limitée.

5 Le contrôle de l'État sur l'école est renforcé sous le roi Reza Shah. À partir de 1928, l'État assure la publication des livres scolaires destinés à l'ensemble des écoles iraniennes. Puis la loi de 1943 rend l'enseignement primaire obligatoire et gratuit, de même qu'elle affirme la responsabilité de l'État pour garantir ces principes. Ces différentes initiatives s'inscrivent dans la volonté politique de renforcer l'État-nation. Non seulement le rôle de l'État s'accroît mais l'école permet aussi une nationalisation des esprits. Les crédits affectés à l'éducation sont augmentés, l'instruction des femmes est encouragée et l'Université de Téhéran créée en 1935. Cependant, le système éducatif reste élitiste et ne peut répondre aux besoins quantitatifs et qualitatifs que nécessite une modernisation économique.

6 Le roi héritier Mohammad Reza Shah tente d'élargir l'accès à l'instruction et encourage notamment le développement de l'enseignement supérieur. Il entreprend la création de nombreuses universités à partir des années 1950 et crée le ministère des sciences et de l'enseignement supérieur en 1968, si bien que l'Iran compte dix-neuf universités nationales en 1978. L'enseignement est aussi encouragé, à un autre niveau, par la mise en place d'efficaces politiques d'alphabétisation et d'accès à l'instruction pour les femmes. 


\section{L'islamisation de l'école}

$7 \mathrm{Au}$ lendemain de la Révolution islamique de 1979, le système éducatif iranien est ouvertement critiqué par le nouveau gouvernement et de nombreux conseils d'experts sont créés pour agir sur ce terrain prioritaire. Khomeyni initie une «islamisation» de l'éducation, mais il n'aspire pas pour autant à un retour vers les établissements religieux traditionnels. Il a l'ambition d'une école à la fois moderne et religieuse, non pervertie par la culture "occidentale » et "coloniale ${ }^{2}$. Face à une école dite inspirée des modèles étrangers, il fait de l'islam la source originelle et légitime de la réflexion sur l'éducation. L'islam est présenté comme la voie de la résistance à la « colonisation » des esprits causée par la politique des rois pahlavis. Les premiers changements passent par la révision des manuels, l'épuration du personnel scolaire, l'abolition de la mixité, la mise en place de nouveaux codes vestimentaires selon les préceptes islamiques (port du voile pour les filles), ou encore l'imposition de pratiques religieuses telles que les prières à midi ou les chants révolutionnaires et religieux dans la journée.

8 Le pouvoir lance ensuite une «révolution culturelle » placée sous la responsabilité du Quartier général de la révolution culturelle créé par Khomeyni en juin 1980. Celle-ci touche en particulier les universités, qui sont fermées. Huit mille professeurs, soit la moitié des effectifs du personnel universitaire en activité à l'époque, sont démis de leurs fonctions et de nouveaux centres de formation sont créés pour former les nouveaux professeurs d'université. De nouveaux responsables sont nommés à la tête des universités qui sont réouvertes à la rentrée 1981. Les orientations majeures du système éducatif de la République islamique sont énoncées dans la loi de 1987 qui décrit le projet de la formation du nouvel homme musulman. «Elle explicite la place de l'homme dans ce monde, le sens de son existence et de son action [...] le rôle de l'école dans la formation religieuse et la socialisation des élèves ${ }^{3}$. Dans son premier article, cette loi revendique une instruction qui repose ouvertement sur le Coran et les textes sacrés. Dans l'article suivant, elle envisage aussi le rôle politique et de socialisation de l'école. Puis dans l'article 4, elle affirme la primauté de la purification sur l'enseignement dans les écoles de la République islamique.

\section{L'organisation scolaire}

\section{L'évolution sous les Pahlavis}

9 Le système scolaire, dans son ensemble, est placé sous la gestion des ministères de l'éducation et de l'enseignement supérieur, créés respectivement dans les années 1920 et 1960. Ces institutions ont pour responsabilité de planifier et de développer les curricula, de réaliser les manuels scolaires, de préparer les professeurs et de définir les examens et les diplômes.

10 L'organisation des curricula et du système scolaire s'est d'abord inspirée du modèle français. Jusqu'au milieu des années 1960, la scolarité se divisait entre les cycles primaire et secondaire d'une durée de six années chacun, et les trois dernières années étaient consacrées à une spécialisation entre trois sections: littérature, sciences et mathématiques. Selon le modèle français, les universités se composent de plusieurs facultés avec différentes filières aux cursus déterminés. La structure de ce système 
scolaire sera critiquée par des conseillers américains et des éducateurs iraniens dans les années 1950. De nouvelles méthodes d'apprentissage verront le jour et poseront les fondements des réformes éducatives réalisées dans les années 1960.

11 Le nouveau système scolaire sera pensé afin de répondre aux besoins d'une économie en développement rapide, et former notamment des techniciens qualifiés. Le cycle primaire, réduit à cinq ans, se consacre alors à l'acquisition de compétences et de connaissances de base et le cursus général est prolongé de trois ans au sein de la nouvelle institution qu'est le collège. Le cycle secondaire, réduit à quatre ans, offre le choix entre une voie académique, préparant aux études supérieures, et une voie professionnelle formant des techniciens. Cette dernière voie de spécialisation ne répondra pas aux attentes de ses concepteurs, qui espéraient voir $40 \%$ des élèves iraniens faire le choix d'un enseignement technique. La voie technique rassemblera d'abord 7,4\% des étudiants en 1976 et jusqu'à 18 \% en 1986 mais les effectifs se ramènent à 15 \% en 1991.

\section{Après la Révolution islamique}

12 La structure et l'organisation du système éducatif sont maintenues sous la République islamique. La scolarité est toujours obligatoire de six à quatorze ans mais, bien que l'enseignement primaire soit généralisé, la proportion des enfants qui bénéficient de l'enseignement obligatoire de huit ans est estimée à $92 \%$ en 2004.

13 Sous la République islamique, la structure du système éducatif est légèrement modifiée dans le secondaire. La distinction entre formations académique et technique est maintenue mais, dans le premier cas, une seule année d'instruction générale précède désormais trois années d'étude en section spécialisée. En 1992, l'enseignement dans les lycées est réduit à trois ans car on crée une année réservée à la préparation des concours d'entrée à l'université. Pour accéder à cette année préparatoire, les élèves doivent avoir une moyenne supérieure à 14 à l'examen correspondant au baccalauréat. De plus, cette année préparatoire est nécessaire pour passer les examens d'entrée dans l'université publique. L'existence de concours à l'entrée de l'université publique en Iran montre l'élitisme de l'enseignement supérieur iranien. La compétition à l'entrée des universités s'explique aussi par le peu de places disponibles.

\section{Les enjeux contemporains}

\section{La féminisation de la population scolaire}

Dans l'école étatisée de la première moitié du $\mathrm{XX}^{\mathrm{e}}$ siècle, le nombre de filles scolarisées augmente rapidement, surtout suite à la loi de 1943 qui rend l'enseignement primaire obligatoire pour les filles comme pour les garçons. La part des filles dans la population scolaire passe ainsi de $27 \%$ en 1943 à $40 \%$ des élèves des écoles primaires en 1978. La même année, elles constituent $37 \%$ des collégiens, $36 \%$ des lycéens et $29 \%$ des étudiants. Après la Révolution islamique, la place des filles dans les institutions scolaires continue de s'accroître et la population féminine se trouve majoritaire dans les universités à partir de la fin des années 1990. Depuis 1997, on compte plus de filles parmi les bacheliers (58\% en 1998) et un nombre plus important d'entre elles remplit les critères scolaires d'admission aux examens d'entrée à l'université. Les filles sont majoritaires, à plus de $60 \%$, dans l'année préparatoire à l'entrée à l'université. Elles connaissent aussi une 
réussite plus marquée dans les concours d'entrée à l'université. De 1998 à 2004, les filles ont obtenu en moyenne $57 \%$ des places offertes par les universités et la progression des filles est également notable dans les domaines traditionnellement réservés aux garçons. En 2004, la proportion des filles s'élève à $25 \%$ dans les facultés d'ingénieurs contre $70 \%$ dans les études médicales, 57 \% en sciences humaines et lettres et $66 \%$ en sciences.

\section{Le défi démographique}

15 L'Iran connaît une croissance démographique impressionnante jusque dans les années 1990 et les effectifs scolaires ne stagnent qu'à partir de 1998. Le nombre d'individus scolarisés passe de 7,8 millions en 1979 à 18 millions en 2001. L'accroissement des effectifs scolaires ne s'explique pas uniquement par la forte croissance de la population iranienne mais aussi par un élargissement de l'accès à l'instruction. Lors de la révolution de 1979, le taux net de scolarisation pour le groupe d'âge 6-10 ans s'élevait à $75 \%$ et en 2004, après plus de vingt ans, $98 \%$ d'entre eux se rendent à l'école. La même année, les taux sont estimées à $88 \%$ pour le collège et $80 \%$ pour le lycée.

L'enseignement supérieur a connu une augmentation frappante du nombre d'étudiants, qui a été multiplié par douze entre 1979 et 2005. La massification de l'enseignement supérieur a été possible grâce à l'Université islamique libre. Créée à partir de 1982, cette université est payante sans être indépendante car elle est placée sous le contrôle des institutions gouvernementales. Elle accueille trois mille étudiants à son ouverture, 176 000 en 1990, et 1,1 million en 2004. Les universités publiques ont également vu leurs effectifs augmenter, passant de 312000 étudiants en 1990 à un peu plus d'un million en 2004. L'université n'est donc plus réservée à une élite mais s'ouvre à un grand nombre de jeunes dans un pays de plus de 70 millions d'habitants où les deux tiers de la population ont moins de trente ans. L'enseignement supérieur en Iran comptait près de 2,1 millions d'étudiants en 2004 contre 175000 en 1979. Au moment de la révolution, il n'existait qu'une vingtaine de sites universitaires implantés uniquement dans les grandes villes, alors que dans les années 1990, le nombre de sites et d'antennes universitaires dépasse la centaine. Malgré ces progrès, l'université ne répond pas à la demande sociale, les places sont limitées à l'université publique et seul un candidat sur dix a pu y trouver une place à la rentrée 2003.

Si le système éducatif a pu répondre à la croissance démographique ainsi qu'aux besoins non satisfaits en matière d'éducation, les dépenses publiques n'ont pas suivi. De 1979 à 1996, les dépenses éducatives ont diminué de $30 \%$. Cet amoindrissement des ressources a un impact sur la qualité du système scolaire. Les infrastructures étant insuffisantes, 50 \% des établissements assurent deux sessions d'enseignement dans la journée et accueillent deux groupes d'élèves, le matin et l'après-midi.

\section{L'enseignement privé}

L'insuffisance structurelle encourage aussi l'émergence d'une privatisation de l'éducation. Face à la difficulté de répondre aux besoins concernant l'éducation, une loi votée en 1988 autorise l'ouverture d'écoles privées. Financées par les ménages avec une aide éventuelle du ministère de l'éducation, ces écoles sont placées sous le contrôle des institutions gouvernementales, suivent le même programme que l'école publique, et les nominations de leurs directeurs sont approuvées par le ministère de l'éducation. Ces 
établissements se développent particulièrement dans les grandes villes et sont destinés aux enfants des familles aisées qui choisissent de financer une instruction privilégiée. Selon le ministère de l'éducation nationale, ces établissements concernaient en 2002 près d'un million de personnes soit $5 \%$ du nombre total des élèves. Ces écoles se concentrent dans les grandes villes, et surtout à Téhéran, la capitale, où elles sont même majoritaires dans les quartiers les plus riches de la ville. Ce sont les élèves issus des établissements privées qui connaissent ensuite les meilleurs taux de réussite aux concours d'entrée des universités publiques. Le renforcement du secteur privé minoritaire entraîne ainsi l'émergence d'un système éducatif à deux vitesses.

\section{BIBLIOGRAPHIE}

Article « éducation », Encyclopaedia Iranica, vol. 8, Costa Mesa, Mazda Publications, 1998.

PAIVANDI S. (2006) : Islam et éducation en Iran. Echec de l'islamisation de l'école en Iran. Paris, L'Harmattan.

\section{NOTES}

1. Un mollah est un docteur en droit coranique.

2. Discours de Khomeyni en 1983, vol. 12, p.177 in PAIVANDI, S., Islam et éducation en Iran. Echec de l'islamisation de l'école en Iran. Paris : L'Harmattan, 2006, p. 72.

3. Ibid., p. 80.

INDEX

Mots-clés : enseignement primaire, enseignement secondaire, système éducatif Index géographique : Iran

\section{AUTEUR}

\section{CLOTILDE REISS}

Master 2, Institut d'études politiques de Lille 